\title{
Nuclear matter properties with nucleon-nucleon forces up to fifth order in the chiral expansion
}

\author{
Jinniu $\mathrm{Hu}^{*}$ \\ School of Physics, Nankai University, Tianjin 300071, China \\ Ying Zhang \\ Department of Physics, School of Science, Tianjin University, Tianjin 300072, China \\ Evgeny Epelbaum \\ Institut für Theoretische Physik II, Ruhr-Universität Bochum, D-44780 Bochum, Germany \\ Ulf-G. Meißner \\ Helmholtz-Institut für Strahlen- und Kernphysik and Bethe Center for Theoretical Physics, Universität Bonn, D-53115 Bonn, Germany \\ and Institut für Kernphysik, Institute for Advanced Simulation and Jülich Center for Hadron Physics, Forschungszentrum Jülich, \\ D-52425 Jülich, Germany \\ Jie Meng \\ School of Physics and State Key Laboratory of Nuclear Physics and Technology, Peking University, Beijing 100871, China; \\ School of Physics and Nuclear Energy Engineering, Beihang University, Beijing 100191, China; \\ and Department of Physics, University of Stellenbosch, Stellenbosch 7602, South Africa \\ (Received 23 January 2017; revised manuscript received 7 August 2017; published 6 September 2017)
}

\begin{abstract}
The properties of nuclear matter are studied using state-of-the-art nucleon-nucleon forces up to fifth order in chiral effective field theory. The equations of state of symmetric nuclear matter and pure neutron matter are calculated in the framework of the Brueckner-Hartree-Fock theory. We discuss in detail the convergence pattern of the chiral expansion and the regulator dependence of the calculated equations of state and provide an estimation of the truncation uncertainty. For all employed values of the regulator, the fifth-order chiral two-nucleon potential is found to generate nuclear saturation properties similar to the available phenomenological high precision potentials. We also extract the symmetry energy of nuclear matter, which is shown to be quite robust with respect to the chiral order and the value of the regulator.
\end{abstract}

DOI: 10.1103/PhysRevC.96.034307

\section{INTRODUCTION}

The nuclear force, a residual strong force between colorless nucleons, lies at the very heart of nuclear physics. Enormous progress has been made towards its quantitative understanding since the seminal work by Yukawa on the one-pion-exchange mechanism, which was published more than eight decades ago [1]. Already in the 1950s, Taketani et al. pointed out that the range of nucleon-nucleon $(N N)$ potential can be divided into three distinct regions [2]. While the long-distance interaction is dominated by one-pion exchange, the two-pion exchange mechanism plays an important role in the intermediate region of $r \sim 1-2 \mathrm{fm}$. Multi-pion exchange interactions are most essential in the core region. After the discovery of heavy mesons, the $N N$ potential was successfully modeled using the one-boson-exchange (OBE) picture $[3,4]$ with multipion exchange potentials being effectively parametrized by single exchanges of heavy mesons such as $\sigma, \omega$, and $\rho$ mesons. With a fairly modest number of adjustable parameters, the OBE potential models such as the Bonn [5,6] and Nijmegen 93 [7] models were able to achieve a semiquantitative description of $N N$ scattering data. Furthermore, based on the general

\footnotetext{
*hujinniu@nankai.edu.cn
}

operator structure of the two-nucleon interaction in coordinate space, a phenomenological $N N$ potential model was also developed by the Argonne group [8]. In the 1990s, highprecision charge-dependent $N N$ potential models such as the Reid93 and Nijmegen I, II [7], AV18 [9], and the CD Bonn [10] potentials were developed, which describe the available proton-proton and neutron-proton elastic scattering data with $\chi^{2} /$ datum $\sim 1$.

While phenomenologically successful, the abovementioned high-precision $N N$ potentials have no clear relation to quantum chromodynamics (QCD), the underlying theory of the strong interactions. Furthermore, they do not provide a straightforward way to generate consistent and systematically improvable many-body forces and exchange currents and do not allow one to estimate the theoretical uncertainty. In this sense, a more promising and systematic approach to nuclear forces and current operators has been proposed by Weinberg in the framework of chiral effective field theory (EFT) based on the most general effective chiral Lagrangian constructed in harmony with the symmetries of QCD [11-13]. The first quantitative studies of $N N$ scattering up to next-to-next-to-leading order $\left(\mathrm{N}^{2} \mathrm{LO}\right)$ in the chiral expansion were carried out by Ordóñez et al. $[14,15]$ using time-ordered perturbation theory; see also $[16,17]$ where the calculations were done using the method of unitary 
transformations. In the early 2000s, the $N N$ potential was worked out to fourth order in the chiral expansion $\left(\mathrm{N}^{3} \mathrm{LO}\right)$ by Epelbaum, Glöckle, and Meißner [18] and by Entem and Machleidt [19] based on the expressions for the pion exchange contributions derived by Kaiser [20-22]. The corresponding three- and four-nucleon forces have also been worked out to $\mathrm{N}^{3} \mathrm{LO}$ [23-27]; see [28,29] for review articles and [30-32] for calculations beyond $\mathrm{N}^{3} \mathrm{LO}$. Recently, fifth-order $\left(\mathrm{N}^{4} \mathrm{LO}\right)$ and even some of the sixth-order contributions to the two-nucleon force were worked out in [33,34], and a new generation of chiral $N N$ potentials up to $\mathrm{N}^{4} \mathrm{LO}$ utilizing a local coordinate-space regulator for the long-range terms was introduced in $[35,36]$. In parallel, a novel simple approach for estimating the theoretical uncertainty from the truncation of the chiral expansion was proposed in [35] and successfully validated for two-nucleon observables $[35,36]$. The algorithm makes use of the explicit knowledge of the contributions to an observable of interest at various orders in the chiral expansion without relying on cutoff variation. The new state-of-the-art $N N$ potentials confirm a good convergence of the chiral expansion for nuclear forces and lead to accurate description of Nijmegen phase shifts [37]. For related recent developments, see Refs. [38,39].

Currently, work is in progress by the recently established Low Energy Nuclear Physics International Collaboration (LENPIC) [40] towards including the consistently regularized three-nucleon force $(3 \mathrm{NF})$ at $\mathrm{N}^{3} \mathrm{LO}$ in $a b$ initio calculations of light- and medium-mass nuclei. In parallel, the novel chiral $N N$ potentials have been tested in nucleon-deuteron elastic scattering and properties of ${ }^{3} \mathrm{H},{ }^{4} \mathrm{He}$, and ${ }^{6} \mathrm{Li}$ [41] and selected electroweak processes [42], where special focus has been put on estimating the theoretical uncertainty at each order of the expansion. These studies have revealed the important role of the $3 \mathrm{NF}$, whose expected contributions to various bound and scattering state observables appear to be in good agreement with the expectation based on the power counting.

Light- and medium-mass nuclei can nowadays be studied using various $a b$ initio methods such as the Green's function Monte Carlo method [43], the self-consistent Green's function method [44], the coupled-cluster approach [45], nuclear lattice simulations [46-48], or the no-core-shell model [49]; see also Ref. [50] for a first application of the relativistic BruecknerHartree-Fock theory to finite nuclei. Infinite nuclear matter has also been widely studied based on various versions of the chiral potentials using, e.g., the quantum Monte Carlo approach [38], self-consistent Green's function method [51,52], the coupledcluster method [53], many-body perturbation theory [54], the functional renormalization group (FRG) method [55,56], and the Brueckner-Hartree-Fock (BHF) theory [57,58]. Recently, Sammarruca et al. discussed the convergence of chiral EFT in infinite nuclear matter using the nonlocal $N N$ potentials up to $\mathrm{N}^{3} \mathrm{LO}[19]$ and including the $3 \mathrm{NF}$ at the $\mathrm{N}^{2} \mathrm{LO}$ (i.e., $Q^{3}$ ) level [59]. Fairly large deviations between the results at different chiral orders as compared with the spread in predictions due to the employed cutoff variation are reported in that paper. This suggests that cutoff variation does not represent a reliable approach to uncertainty quantification, which is fully in line with the conclusions of [35]. Regulator artifacts in uniform matter have also been addressed in Ref. [60]. For a different power counting, that explicitly accounts for the scale set by the Fermi momentum and that also describes pure neutron matter (PNM) and symmetric nuclear matter (SNM) well, see Ref. [61].

In this work, we calculate, for the first time, the properties of SNM and PNM based on the latest generation of chiral $N N$ potentials up to $\mathrm{N}^{4} \mathrm{LO}$ of Refs. [35,36] using the BHF theory. The purpose of our study is twofold. First, we explore the performance of the new generation of the chiral forces in microscopic calculations of the equations of state (EOS) of SNM and PNM. This will allow one to draw indirect conclusions on the expected size of the contributions due to many-body forces. Second, by performing an error analysis along the lines of Refs. $[35,36,41]$ without relying on cutoff variation, we estimate the theoretical accuracy in the description of the nuclear EOS achievable at various orders of the chiral expansion.

\section{THE EQUATIONS OF STATE OF NUCLEAR MATTER}

The details of BHF theory for nuclear matter can be found in Refs. [57,62]. In Fig. 1, we show our results for the density dependence of the energy per nucleon of symmetric nuclear matter and pure neutron matter for all available chiral orders and cutoff values, where the $G$ matrices are solved up to the partial waves $J=6$. We remind the reader that the longrange contributions are regularized in the newest chiral $N N$ potentials by multiplying the corresponding coordinate-space expressions with the function

$$
f(r)=\left[1-\exp \left(-\frac{r^{2}}{R^{2}}\right)\right]^{n}, \quad n=6, R=0.8-1.2 \mathrm{fm} .
$$

For contact interactions, a nonlocal Gaussian regulator in momentum space is employed with the cutoff $\Lambda$ being related to $R$ via $\Lambda=2 / R$. We emphasize that the calculations reported in this paper do not include the contributions of three- and four-nucleon forces and are thus incomplete starting from $\mathrm{N}^{2} \mathrm{LO}$.

For SNM, the LO (i.e., $Q^{0}$ ), NLO (i.e., $Q^{2}$ ), and $\mathrm{N}^{4} \mathrm{LO} N N$ potentials yield larger binding energies for softer interactions (i.e., for larger cutoffs $R$ ), while the situation is opposite at $\mathrm{N}^{2} \mathrm{LO}$ and $\mathrm{N}^{3} \mathrm{LO}$. For PNM, the harder (softer) interactions yield more (less) attraction at $\mathrm{LO}, \ldots, \mathrm{N}^{3} \mathrm{LO}\left(\mathrm{N}^{4} \mathrm{LO}\right)$. This complicated pattern suggests that the EOS is rather sensitive to the details of the nuclear force and especially to the interplay between its intermediate and short-range components. Given that the potentials at NLO and $\mathrm{N}^{2} \mathrm{LO}$ as well as at $\mathrm{N}^{3} \mathrm{LO}$ and $\mathrm{N}^{4} \mathrm{LO}$ involve the same set of (isospin-invariant) contact interactions, these changes in the pattern of the $R$ dependence of the calculated energies from $\mathrm{N}^{3} \mathrm{LO}$ to $\mathrm{N}^{4} \mathrm{LO}$ and, in the case of SNM, also from NLO to $\mathrm{N}^{2} \mathrm{LO}$ reflect the impact of the two-pion exchange (TPE) contributions at $\mathrm{N}^{2} \mathrm{LO}$ and $\mathrm{N}^{4}$ LO. These findings are in line with the ones of Ref. [63], where the important role of the TPE for nuclear binding was conjectured. Our results at NLO agree well with the 

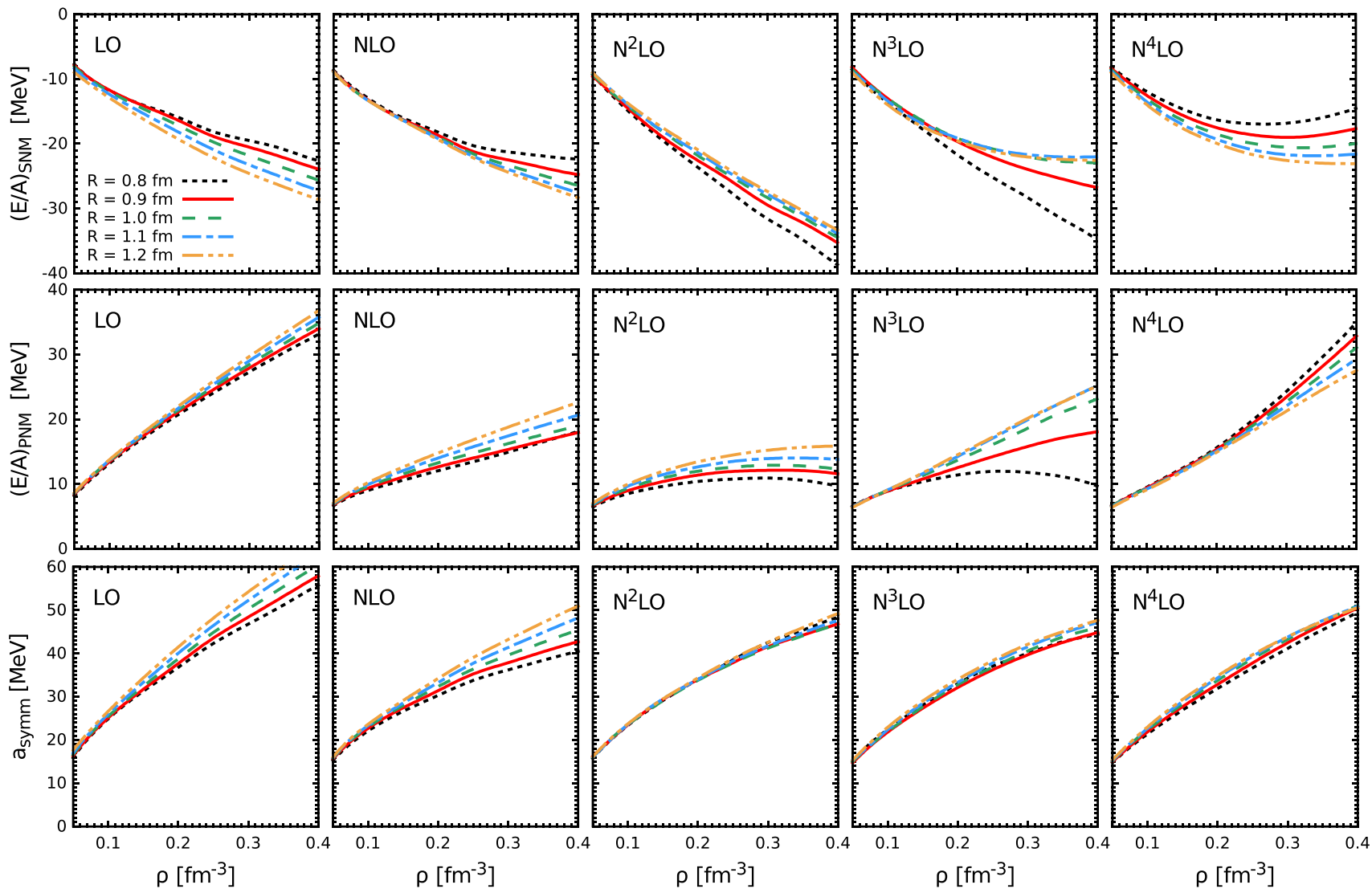

FIG. 1. Density dependence of the energy per particle of SNM $(E / A)_{\mathrm{SNM}}$ (upper row), of PNM $(E / A)_{\mathrm{PNM}}$ (middle row), and of the symmetry energy $a_{\text {symm }}$ (lower row) based on chiral $N N$ potentials of [35,36] for all available cutoff values in the range of $R=0.8-1.2$ fm.

ones reported in [59] both for $\mathrm{SNM}$ and $\mathrm{PNM}^{1}$ and with the quantum Monte Carlo calculation of Ref. [38] for PNM. For example, at the saturation density of $\rho=0.16 \mathrm{fm}^{-3}$, the authors of Ref. [59] found at NLO for the employed cutoff range the values of $E / A=-21$ to $-17 \mathrm{MeV}$ for SNM and $E / A=10$ to $12 \mathrm{MeV}$ for PNM, which has to be compared with our NLO results of $E / A=-17$ to $-16 \mathrm{MeV}$ for SNM and $E / A=11$ to $13 \mathrm{MeV}$ for PNM. The NLO prediction of Ref. [38] for the energy per particle of PNM at $\rho=0.15 \mathrm{fm}^{-3}$ is $E / A=10$ to $13 \mathrm{MeV}$. Interestingly, the cutoff dependence of the energy per particle of PNM at NLO is qualitatively different from the one found in [59], which demonstrates that the form of the regulator does significantly affect the properties of the resulting potentials.

Generally, our results for both SNM and PNM show an increasing attraction in the $N N$ force when going from LO to $\mathrm{N}^{2} \mathrm{LO}$, that can probably be traced back to the two-pion exchange potential (TPEP), which has a very strong attractive central isoscalar piece. At $\mathrm{N}^{3} \mathrm{LO}$, the chiral TPEP receives further attractive contributions but also develops a repulsive short-range core. The additional repulsion at $\mathrm{N}^{4} \mathrm{LO}$ comes from the contributions to the TPEP at this order. The EOSs

\footnotetext{
${ }^{1}$ We cannot compare our $\mathrm{N}^{2} \mathrm{LO}$ and $\mathrm{N}^{3} \mathrm{LO}$ predictions with those of Ref. [59] since no results based on $N N$ interactions only are provided in that work.
}

based on the $\mathrm{N}^{3} \mathrm{LO}$ and $\mathrm{N}^{4} \mathrm{LO}$ potentials alone show saturation points below $\rho=0.4 \mathrm{fm}^{-3}$ except for $\mathrm{N}^{3} \mathrm{LO}$ at $R=0.8 \mathrm{fm}$ and $R=0.9 \mathrm{fm}$.

It is instructive to compare the results based on the most accurate chiral potentials at $\mathrm{N}^{4} \mathrm{LO}$ with the ones from highprecision phenomenological interactions such as the AV18 potential [9]. In Table I, we list the saturation properties, saturation densities, and saturation binding energies per particle, and the nonrelativistic effective mass of the nucleon [64] at the saturation point for the AV18 and $\mathrm{N}^{4} \mathrm{LO}$ potentials. Notice that the listed saturation properties are still far from the empirical data $\left(\rho_{\text {sat }} \sim 0.16 \mathrm{fm}^{-3}\right.$ and $\left.E / A \sim 16 \mathrm{MeV}\right)$ due to the missing $3 \mathrm{NF}$ contributions [57,62]. Naturally, we observe that the results based on the hardest version of the $\mathrm{N}^{4} \mathrm{LO}$ potential with $R=0.8 \mathrm{fm}$ are rather similar to those based on AV18. In Table II, the partial wave contributions to potential energy at the empirical saturation density $\rho=0.16 \mathrm{fm}^{-3}$ for different $N N$ potentials are listed from ${ }^{1} S_{0}$ to ${ }^{3} F_{3}$ states. It is found that all contributions are nearly cutoff independent expect the ones from ${ }^{1} S_{0},{ }^{3} S_{1}{ }^{3} D_{1}$, and ${ }^{3} D_{3^{-}}{ }^{3} G_{3}$ states, which are decreasing with the cutoffs $R$. Actually, the size of these contributions is strongly dependent on the central and tensor components in the $N N$ potential. The smaller cutoff $R$ corresponds to harder interactions and gives more repulsive contribution to the $N N$ potential at short distance. It leads to smaller binding energy. Our results for the saturation density and binding energy confirm the linear correlation between these two quantities, 
TABLE I. Saturation properties of SNM based on the AV18 potential and the $\mathrm{N}^{4} \mathrm{LO}$ chiral $N N$ potentials for all available cutoff values.

\begin{tabular}{lrrrrr}
\hline \hline & $\mathrm{AV} 18$ & $\mathrm{~N}^{4} \mathrm{LO}_{R=0.8 \mathrm{fm}}$ & $\mathrm{N}^{4} \mathrm{LO}_{R=0.9 \mathrm{fm}}$ & $\mathrm{N}^{4} \mathrm{LO}_{R=1.0 \mathrm{fm}}$ & $\mathrm{N}^{4} \mathrm{LO}_{R=1.1 \mathrm{fm}}$ \\
\hline$\rho_{\text {sat }}\left(\mathrm{fm}^{-3}\right)$ & 0.26 & 0.28 & 0.29 & 0.31 & 0.35 \\
$E / A(\mathrm{MeV})$ & -17.78 & -17.14 & -19.15 & -20.67 & -21.92 \\
$M^{*} / M$ & 0.71 & 0.74 & 0.73 & 0.72 & 0.70 \\
\hline \hline
\end{tabular}

known as the Coester line [65]; see also [57]. Calculations within the BHF theory using phenomenological potentials have revealed that the position on the Coester line is correlated with the deuteron $D$-state probability $P_{D}$, with smaller values of $P_{D}$ typically resulting in smaller saturation energy and density $[6,57]$. We observe the opposite trend for the chiral $\mathrm{N}^{4} \mathrm{LO}$ potentials with $P_{D}=4.28 \%, 4.29 \%, 4.40 \%, 4.74 \%$, and $5.12 \%$ for $R=0.8 \mathrm{fm}$ to $R=1.2 \mathrm{fm}$, respectively. This is similar to the lack of correlation between $P_{D}$ and the triton binding energy for the novel chiral potentials [41].

We have also extracted the symmetry energy of nuclear matter $a_{\text {symm }}(\rho)$, which is defined in terms of the expansion of the asymmetric nuclear matter in powers of the asymmetry parameter $\delta \equiv\left(\rho_{n}-\rho_{p}\right) / \rho$, with $\rho_{n}$ and $\rho_{p}$ referring to the neutron and proton number densities via

$$
\frac{E}{A}(\rho, \delta)=\frac{E}{A}(\rho, 0)+a_{\text {symm }}(\rho) \delta^{2}+\cdots .
$$

The terms beyond the quadratic one are known to be very small [66], so that the symmetry energy can be well approximated by

$$
a_{\mathrm{symm}}(\rho)=\left(\frac{E}{A}\right)_{\mathrm{PNM}}-\left(\frac{E}{A}\right)_{\mathrm{SNM}},
$$

where $E / A$ is viewed as a function of $\rho$ and $\delta$. While the calculated symmetry energies show significant cutoff dependence at LO and NLO, which is comparable to that of $(E / A)_{\mathrm{SNM}}$ and $(E / A)_{\mathrm{PNM}}$, the results at higher orders are almost insensitive to the values of $R$ and show a little variation with the order of the chiral expansion. The resulting value of $a_{\text {symm }}=27.9-30.5 \mathrm{MeV}$ at the empirical saturation density, calculated using the $\mathrm{N}^{4} \mathrm{LO}$ potentials, is consistent with the empirical constraints and the results from the phenomenological high-precision $N N$ potentials [57] with $a_{\text {symm }}=28.5-32.6 \mathrm{MeV}$ at $\rho=0.17 \mathrm{fm}^{-3}$ and the ones from the functional renormalization group method with $a_{\text {symm }}=$ 29.0-33.0 MeV at $\rho=0.16 \mathrm{fm}^{-3}$ [55]. Furthermore, Vidaña et al. also studied the properties of the symmetry energy with the AV18 potential plus a phenomenological three-body force of Urbana type [67]. However, it is found that the isovector properties of nuclear matter are not affected by the three-body force too much; just a few $\mathrm{MeV}$ on symmetry energy as shown in Ref. [68].

\section{UNCERTAINTY QUANTIFICATION}

We now turn to the important question of uncertainty quantification from the truncation of the chiral expansion. Actually, Baldo et al. attempted to quantify the theoretical uncertainties of the EOSs with the family of Argonne $N N$ potentials by comparing the BHF theory to other many-body approaches [69]. These uncertainties are strongly dependent on the methodologies of nuclear many-body approximation to treat the spin structures of potentials. Here we follow the approach formulated in Ref. [35], which makes use of the explicitly known contributions to an observable of interest at various chiral orders to estimate the size of truncated terms without relying on cutoff variation. This approach is applicable to any observable of interest provided one can estimate the typical momentum scale $p$ involved in a process, which governs the expansion parameter $Q \in\left\{p / \Lambda_{\mathrm{b}}, M_{\pi} / \Lambda_{\mathrm{b}}\right\}$. Here, $M_{\pi}$ is the pion mass while $\Lambda_{\mathrm{b}}$ refers to the breakdown scale of the chiral expansion. The scale $p$ is not to be confused with the highest integration momenta when calculating the scattering amplitude, which are set by the employed ultraviolet cutoff. Rather, $p$ is to be viewed as an effective momentum of the nucleons after renormalizing the amplitude. For scattering observables, $p$ is naturally set by the external center-of-mass

TABLE II. Contributions of the various partial waves (in units of MeV) to the binding energies of SNM at the empirical saturation density,

\begin{tabular}{|c|c|c|c|c|c|c|}
\hline & AV18 & $\mathrm{N}^{4} \mathrm{LO}_{R=0.8 \mathrm{fm}}$ & $\mathrm{N}^{4} \mathrm{LO}_{R=0.9 \mathrm{fm}}$ & $\mathrm{N}^{4} \mathrm{LO}_{R=1.0 \mathrm{fm}}$ & $\mathrm{N}^{4} \mathrm{LO}_{R=1.1 \mathrm{fm}}$ & $\mathrm{N}^{4} \mathrm{LO}_{R=1.2 \mathrm{fm}}$ \\
\hline${ }^{1} S_{0}$ & -15.01 & -14.32 & -14.83 & -15.19 & -15.47 & -15.81 \\
\hline${ }^{3} P_{0}$ & -3.07 & -3.17 & -3.17 & -3.18 & -3.18 & -3.18 \\
\hline${ }^{3} P_{1}$ & 8.47 & 9.16 & 9.17 & 9.14 & 9.15 & 9.14 \\
\hline${ }^{1} P_{1}$ & 3.36 & 3.61 & 3.59 & 3.57 & 3.56 & 3.55 \\
\hline${ }^{3} P_{2}-{ }^{3} F_{2}$ & -6.89 & -7.71 & -7.71 & -7.73 & -7.74 & -7.79 \\
\hline${ }^{3} D_{2}$ & -3.34 & -3.65 & -3.65 & -3.66 & -3.67 & -3.68 \\
\hline${ }^{3} D_{3}-{ }^{3} G_{3}$ & 0.08 & 0.20 & 0.19 & 0.16 & 0.13 & 0.09 \\
\hline${ }^{1} F_{3}$ & 0.66 & 0.72 & 0.72 & 0.72 & 0.72 & 0.72 \\
\hline${ }^{3} F_{3}$ & 1.19 & 1.31 & 1.30 & 1.30 & 1.30 & 1.29 \\
\hline
\end{tabular}
$\rho=0.16 \mathrm{fm}^{-3}$, for the AV18 and chiral $\mathrm{N}^{4} \mathrm{LO} N N$ potentials for all available cutoff values. 

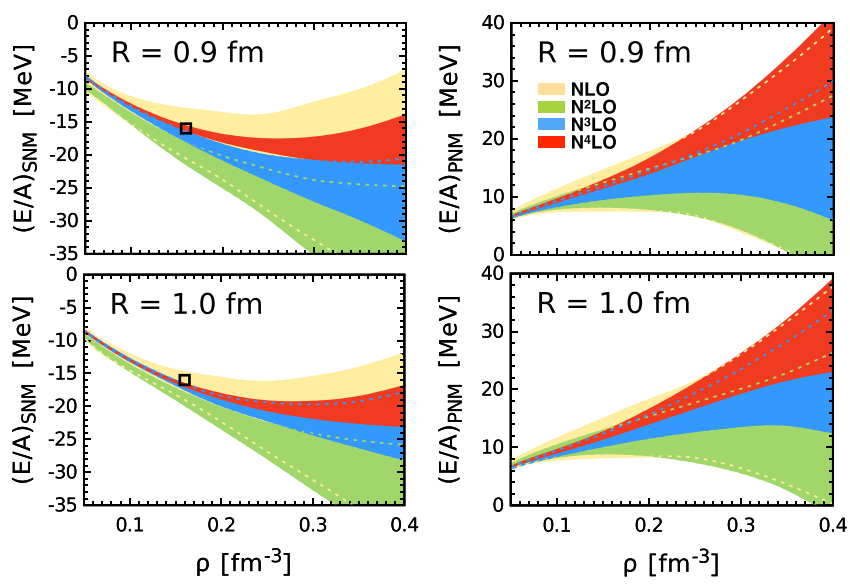

FIG. 2. Predictions for the EOS of SNM (left column) and PNM (right column) based on the chiral $N N$ potentials of Refs. [35,36] for $R=0.9 \mathrm{fm}$ (upper row) and $R=1.0 \mathrm{fm}$ (lower row) along with the estimated theoretical uncertainties. Open rectangles visualize the empirical saturation point of symmetric nuclear matter.

momentum [35]. It is less obvious how to estimate the momentum scale $p$ for finite nuclei. In [41], the expansion parameter for light nuclei was assumed to be $Q=M_{\pi} / \Lambda_{\mathrm{b}}$. On the other hand, in heavy nuclei one expects the scale $p$ to increase as a consequence of the Pauli principle. For infinite nuclear matter, it seems most natural to estimate $p$ by the corresponding Fermi momentum, which is directly related to the density and sets the inverse distance scale in the system. The validity of such an estimation may eventually be tested within a Bayesian approach along the lines of Refs. [70]. Such an analysis, however, goes beyond the scope of our work. Here and in what follows, we assume $p$ to be given by the corresponding Fermi momentum.

The algorithm proposed in [35] has been adjusted in Ref. [41] to enable applications to incomplete few- and manynucleon calculations based on two-nucleon forces only. Here and in what follows, we use the method as formulated in that paper, which was also employed in [42]. The breakdown scale of the nuclear chiral EFT was estimated to be $\Lambda_{b} \simeq 600 \mathrm{MeV}$ [35]. ${ }^{2}$ The Bayesian analysis of the chiral EFT predictions for the $N N$ total cross section of Ref. [70] has revealed that the actual breakdown scale may even be a little higher than $\Lambda_{\mathrm{b}} \simeq 600 \mathrm{MeV}$ for $R=0.9 \mathrm{fm}$.

In Fig. 2, we show the results for the EOS for SNM and PNM, including the estimated theoretical uncertainties at various orders of the chiral expansion for the most accurate versions of the $N N$ potentials with $R=0.9 \mathrm{fm}$ and $R=$ $1.0 \mathrm{fm}[35,36]$. The expansion parameter $Q$ at a given density is estimated by identifying the momentum scale $p$ with the Fermi momentum $k_{\mathrm{F}}$, which is related to the density $\rho$ via $\rho=$ $2 k_{\mathrm{F}}^{3} /\left(3 \pi^{2}\right)\left[\rho=k_{\mathrm{F}}^{3} /\left(3 \pi^{2}\right)\right]$ for SNM (PNM), and assuming

\footnotetext{
${ }^{2}$ To account for increasing finite-cutoff artifacts using softer versions of the chiral forces, the lower values of $\Lambda_{b}=500$ and $400 \mathrm{MeV}$ were employed in calculations based on $R=1.1 \mathrm{fm}$ and $R=1.2$ fm, respectively.
}

$\Lambda_{\mathrm{b}}=600 \mathrm{MeV}$. At the saturation density, the achievable accuracy of the chiral EFT predictions for the energy per particle may be expected to be about $\pm 1.5 \mathrm{MeV}( \pm 0.3 \mathrm{MeV})$ for $\mathrm{SNM}$ and $\pm 2 \mathrm{MeV}( \pm 0.7 \mathrm{MeV})$ for PNM at $\mathrm{N}^{2} \mathrm{LO}\left(\mathrm{N}^{4} \mathrm{LO}\right)$. Notice that the expected accuracy at $\mathrm{N}^{4} \mathrm{LO}$ is significantly smaller than the current model dependence for these quantities. We further emphasize that the presented estimations should be taken with some care due to the nonavailability of complete calculations beyond NLO. More reliable estimations of the theoretical uncertainty using the approach of [35] will be possible once the corresponding three- and four-nucleon forces are included. Furthermore, we also do not consider the uncertainty associated with the approximations from the BHF theory in this work.

Our results confirm the conclusions of [59] that cutoff variation does not provide an adequate way for estimating the uncertainties in the calculations of the nuclear EOS. As discussed in [35], the residual cutoff dependence of observables may generally be expected to underestimate the theoretical uncertainty at NLO and $\mathrm{N}^{3} \mathrm{LO}$, which is consistent with our results. Further, the spread of results for different values of $R$ at $\mathrm{N}^{4} \mathrm{LO}$ at nuclear saturation density is about $0.3 \mathrm{MeV}(0.7 \mathrm{MeV})$ for SNM (PNM), which is similar to the estimated uncertainty at this order. However, we refrain from drawing more definite conclusions on the cutoff dependence based on the incomplete calculations.

Finally, we have also quantified the achievable accuracy of the theoretical determination of the symmetry energy $a_{\text {symm }}$ and the slope parameter $L$, defined as $L=3 \rho \partial(E / A)_{\mathrm{SNM}} / \partial \rho$, at the empirical saturation density. These important quantities have been constrained by the available experimental information on, e.g., neutron skin thickness, heavy ion collisions, and dipole polarizabilities leading to the ranges of $29 \lesssim a_{\text {symm }} \lesssim$ $33 \mathrm{MeV}$ and $40 \lesssim L \lesssim 62 \mathrm{MeV}$ [71-73]. In Fig. 3, we show our results for these quantities using the $N N$ potentials from $\mathrm{LO}$ to $\mathrm{N}^{4} \mathrm{LO}$ along with the estimated theoretical uncertainties. Especially for the slope parameter, a complete calculation at $\mathrm{N}^{4} \mathrm{LO}$ would yield a theoretical prediction with high accuracy.

\section{SUMMARY AND CONCLUSIONS}

In summary, we calculated the equations of state (EOSs) of SNM and PNM with the state-of-the-art chiral $N N$ potentials from LO to $\mathrm{N}^{4} \mathrm{LO}$ in the framework of Brueckner-HartreeFock theory. At $\mathrm{N}^{4} \mathrm{LO}$, the EOS of SNM has saturation points for all employed cutoff values, with the corresponding saturation densities and binding energies per particle being within the ranges 0.28 to $0.40 \mathrm{fm}^{-3}$ and -17.14 to $-23.28 \mathrm{MeV}$, respectively. These values are compatible with the ones based on the phenomenological high-precision potentials such as the AV18 potential. The symmetry energy and the slope parameter at the saturation density are found to be in the ranges $a_{\text {symm }}=27.9-30.5 \mathrm{MeV}$ and $L=49.4-55.0 \mathrm{MeV}$, respectively, using the $\mathrm{N}^{4} \mathrm{LO}$ potentials with the cutoff in the range $R=0.8-1.2 \mathrm{fm}$.

We have also estimated the achievable theoretical accuracy at various orders in the chiral expansion using the novel approach formulated in Refs. [35,41] and discussed the convergence of the chiral expansion. Similar to [59], we find 

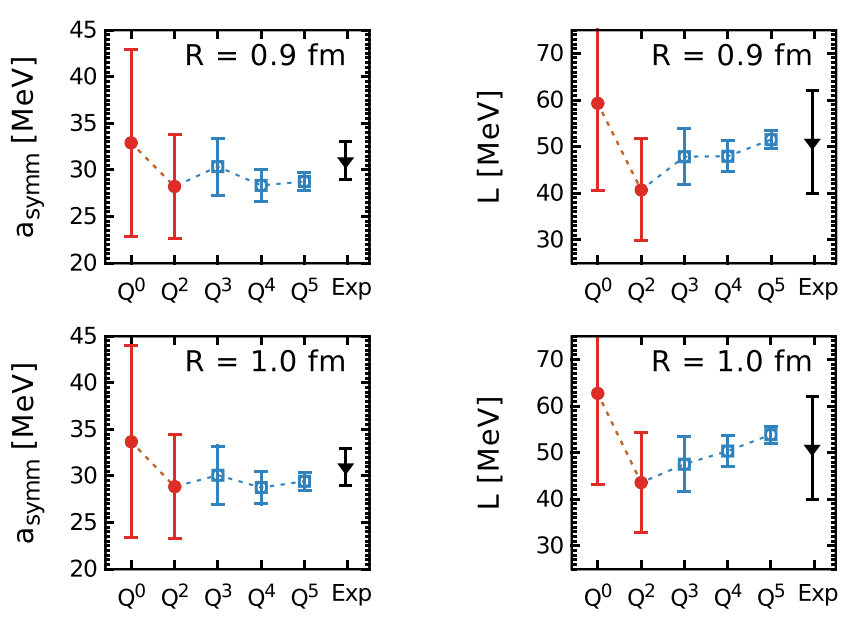

FIG. 3. Chiral expansion of the symmetry energy $a_{\text {symm }}$ (left panel) and the slope parameter $L$ (right panel) at the empirical saturation density $\rho=0.16 \mathrm{fm}^{-3}$ for the cutoff values $R=0.9 \mathrm{fm}$ (upper row) and $R=1.0 \mathrm{fm}$ (lower row) along with the estimated theoretical uncertainty. Solid circles (open rectangles) show the complete results at a given chiral order (incomplete results based on $N N$ interactions only). Solid triangles show the current experimental constraints on $a_{\text {symm }}$ and $L$ as described in the text.

that the residual cutoff dependence of the energy per particle does not allow for a reliable estimation of the theoretical uncertainty; see also the discussion in Ref. [35]. Although, there are still many open questions, such as the sensitivity of EOS on the cutoff regularizations, the renormalization of $N N$ potential, the role and importance of many-body forces, and so on, chiral EFT may be expected to provide an accurate description of SNM and PNM at the saturation density, with the expected accuracy of a few percent on binding energy at $\mathrm{N}^{4} \mathrm{LO}$. At this order, a semiquantitative description of the EOS should be possible up to about twice the saturation density of nuclear matter, which is limited by the available cutoff values. Clearly, this will require a consistent inclusion of the corresponding many-body forces. Work along these lines is in progress to compare with the existing calculations with two-body and three-body chiral force [52,59].

\section{ACKNOWLEDGMENTS}

We would like to thank Arnau Rios Huguet for sharing his insights into the topics discussed here. U.G.M. thanks the ITP (CAS, Beijing) for hospitality, where part of this work was done. This work was supported in part by the National Natural Science Foundation of China (Grants No. 11335002, No. 11405090, No. 11405116, and No. 11621131001), DFG (SFB/TR 110, "Symmetries and the Emergence of Structure in QCD") and BMBF (Contract No. 05P2015-NUSTAR R\&D). The work of U.G.M. was supported in part by The Chinese Academy of Sciences (CAS) President's International Fellowship Initiative (PIFI) Grant No. 2015VMA076.
[1] H. Yukawa, Proc. Phys. Math. Soc. Jpn. 17, 48 (1935).

[2] M. Taketani, S. Nakamura, and M. Sasaki, Prog. Theor. Phys. 6, 581 (1951).

[3] P. Signell, Adv. Nucl. Phys. 2, 223 (1969).

[4] K. Erkelenz, Phys. Rep. 13, 191 (1974).

[5] R. Machleidt, K. Holinde, and C. Elster, Phys. Rep. 149, 1 (1987).

[6] R. Machleidt, Adv. Nucl. Phys. 19, 189 (1989).

[7] V. G. J. Stoks, R. A. M. Klomp, C. P. F. Terheggen, and J. J. de Swart, Phys. Rev. C 49, 2950 (1994).

[8] R. B. Wiringa, R. A. Smith, and T. L. Ainsworth, Phys. Rev. C 29, 1207 (1984).

[9] R. B. Wiringa, V. G. J. Stoks, and R. Schiavilla, Phys. Rev. C 51, 38 (1995).

[10] R. Machleidt, Phys. Rev. C 63, 024001 (2001).

[11] S. Weinberg, Phys. Lett. B 251, 288 (1990).

[12] S. Weinberg, Nucl. Phys. B 363, 3 (1991).

[13] S. Weinberg, Phys. Lett. B 295, 114 (1992).

[14] C. Ordóñez, L. Ray, and U. van Kolck, Phys. Rev. Lett. 72, 1982 (1994).

[15] C. Ordóñez, L. Ray, and U. van Kolck, Phys. Rev. C 53, 2086 (1996).

[16] E. Epelbaum, W. Glöckle, and U.-G. Meißner, Nucl. Phys. A 637, 107 (1998).

[17] E. Epelbaum, W. Glöckle, and U.-G. Meißner, Nucl. Phys. A 671, 295 (2000).

[18] E. Epelbaum, W. Glöckle, and U.-G. Meißner, Nucl. Phys. A 747, 362 (2005).
[19] D. R. Entem and R. Machleidt, Phys. Rev. C 68, 041001 (2003)

[20] N. Kaiser, Phys. Rev. C 62, 024001 (2000).

[21] N. Kaiser, Phys. Rev. C 61, 014003 (1999).

[22] N. Kaiser, Phys. Rev. C 64, 057001 (2001).

[23] S. Ishikawa and M. R. Robilotta, Phys. Rev. C 76, 014006 (2007).

[24] V. Bernard, E. Epelbaum, H. Krebs, and U.-G. Meißner, Phys. Rev. C 77, 064004 (2008).

[25] V. Bernard, E. Epelbaum, H. Krebs, and U.-G. Meißner, Phys. Rev. C 84, 054001 (2011).

[26] E. Epelbaum, Phys. Lett. B 639, 456 (2006).

[27] E. Epelbaum, Eur. Phys. J. A 34, 197 (2007).

[28] E. Epelbaum, H. W. Hammer, and U.-G. Meißner, Rev. Mod. Phys. 81, 1773 (2009).

[29] R. Machleidt and D. R. Entem, Phys. Rep. 503, 1 (2011).

[30] H. Krebs, A. Gasparyan, and E. Epelbaum, Phys. Rev. C 85, 054006 (2012).

[31] H. Krebs, A. Gasparyan, and E. Epelbaum, Phys. Rev. C 87, 054007 (2013).

[32] L. Girlanda, A. Kievsky, and M. Viviani, Phys. Rev. C 84, 014001 (2011).

[33] D. R. Entem, N. Kaiser, R. Machleidt, and Y. Nosyk, Phys. Rev. C 91, 014002 (2015).

[34] D. R. Entem, N. Kaiser, R. Machleidt, and Y. Nosyk, Phys. Rev. C 92, 064001 (2015).

[35] E. Epelbaum, H. Krebs, and U.-G. Meißner, Eur. Phys. J. A 51, 53 (2015). 
[36] E. Epelbaum, H. Krebs, and U.-G. Meißner, Phys. Rev. Lett. 115, 122301 (2015).

[37] V. G. J. Stoks, R. A. M. Klomp, M. C. M. Rentmeester, and J. J. de Swart, Phys. Rev. C 48, 792 (1993).

[38] A. Gezerlis, I. Tews, E. Epelbaum, S. Gandolfi, K. Hebeler, A. Nogga, and A. Schwenk, Phys. Rev. Lett. 111, 032501 (2013).

[39] M. Piarulli, L. Girlanda, R. Schiavilla, R. N. Perez, J. E. Amaro, and E. R. Arriola, Phys. Rev. C 91, 024003 (2015).

[40] See http://www. lenpic.org

[41] S. Binder et al. (LENPIC Collaboration), Phys. Rev. C 93, 044002 (2016).

[42] R. Skibinski, J. Golak, K. Topolnicki, H. Witaßa, E. Epelbaum, H. Krebs, H. Kamada, U.-G. Meißner, and A. Nogga, Phys. Rev. C 93, 064002 (2016).

[43] J. Carlson, S. Gandolfi, F. Pederiva, S. C. Pieper, R. Schiavilla, K. E. Schmidt, and R. B. Wiringa, Rev. Mod. Phys. 87, 1067 (2015).

[44] W. H. Dickhoff and C. Barbieri, Prog. Part. Nucl. Phys. 52, 377 (2004).

[45] G. Hagen, T. Papenbrock, M. Hjorth-Jensen, and D. J. Dean, Rep. Prog. Phys. 77, 096302 (2014).

[46] D. Lee, Prog. Part. Nucl. Phys. 63, 117 (2009).

[47] E. Epelbaum, H. Krebs, T. A. Lähde, D. Lee, U.-G. Meißner, and G. Rupak, Phys. Rev. Lett. 112, 102501 (2014).

[48] U.-G. Meißner, Nucl. Phys. News. 24, 11 (2014).

[49] B. R. Barrett, P. Navratil, and J. P. Vary, Prog. Part. Nucl. Phys. 69, 131 (2013).

[50] S. H. Shen, J. N. Hu, H. Z. Liang, J. Meng, P. Ring, and S. Q. Zhang, Chin. Phys. Lett. 33, 102103 (2016).

[51] A. Carbone, A. Polls, and A. Rios, Phys. Rev. C 88, 044302 (2013).

[52] A. Carbone, A. Rios, and A. Polls, Phys. Rev. C 90, 054322 (2014).

[53] G. Hagen, T. Papenbrock, A. Ekström, K. A. Wendt, G. Baardsen, S. Gandolfi, M. Hjorth-Jensen, and C. J. Horowitz, Phys. Rev. C 89, 014319 (2014).

[54] C. Drischler, V. Somà, and A. Schwenk, Phys. Rev. C 89, 025806 (2014).
[55] M. Drews and W. Weise, Phys. Rev. C 91, 035802 (2015).

[56] M. Drews and W. Weise, Prog. Part. Nucl. Phys. 93, 69 (2017).

[57] Z. H. Li, U. Lombardo, H.-J. Schulze, W. Zuo, L. W. Chen, and H. R. Ma, Phys. Rev. C 74, 047304 (2006).

[58] R. Machleidt, P. Liu, D. R. Entem, and E. R. Arriola, Phys. Rev. C 81, 024001 (2010).

[59] F. Sammarruca, L. Coraggio, J. W. Holt, N. Itaco, R. Machleidt, and L. E. Marcucci, Phys. Rev. C 91, 054311 (2015).

[60] A. Dyhdalo, R. J. Furnstahl, K. Hebeler, and I. Tews, Phys. Rev. C 94, 034001 (2016).

[61] A. Lacour, J. A. Oller, and U.-G. Meißner, Ann. Phys. (NY) 326, 241 (2011).

[62] M. Baldo and C. Maieron, J. Phys. G 34, R243 (2007).

[63] S. Elhatisari, N. Li, A. Rokash, J. M. Alarcon, D. Du, N. Klein, B. N. Lu, U.-G. Meissner, E. Epelbaum, H. Krebs, T. A. Lahde, D. Lee, and G. Rupak, Phys. Rev. Lett. 117, 132501 (2016).

[64] A. Li, J. N. Hu, X. L. Shang, and W. Zuo, Phys. Rev. C 93, 015803 (2016).

[65] F. Coester, S. Cohen, B. Day, and C. M. Vincent, Phys. Rev. C 1, 769 (1970).

[66] C. Drischler, K. Hebeler, and A. Schwenk, Phys. Rev. C 93, 054314 (2016).

[67] I. Vidaña, C. Providência, A. Polls, and A. Rios, Phys. Rev. C 80, 045806 (2009).

[68] D. Logoteta, I. Bombaci, and A. Kievsky, Phys. Lett. B 758, 449 (2016).

[69] M. Baldo, A. Polls, A. Rios, H.-J. Schulze, and I. Vidaña, Phys. Rev. C 86, 064001 (2012).

[70] R. J. Furnstahl, N. Klco, D. R. Phillips, and S. Wesolowski, Phys. Rev. C 92, 024005 (2015).

[71] M. B. Tsang, J. R. Stone, F. Camera, P. Danielewicz, S. Gandolfi, K. Hebeler, C. J. Horowitz, J. Lee, W. G. Lynch, Z. Kohley, R. Lemmon, P. Moller, T. Murakami, S. Riordan, X. Roca-Maza, F. Sammarruca, A. W. Steiner, I. Vidana, and S. J. Yennello, Phys. Rev. C 86, 015803 (2012).

[72] J. M. Lattimer and Y. Lim, Astrophys. J. 771, 51 (2013).

[73] J. M. Lattimer and A. W. Steiner, Eur. Phys. J. A 50, 40 (2014). 